\title{
Intraoperative Nursing Care of Patients Undergoing Transcatheter Aortic Valve Replacement via Femoral Artery Approach
}

\author{
Xuanjing Li ${ }^{1}$, Shengqi Wang ${ }^{2}$, Fan Yang ${ }^{1, ~ *, ~ J i a n ~ Y u ~}{ }^{1}$, Xianzhen Huang ${ }^{1}$, Wenying $\mathrm{Li}^{1}$ \\ ${ }^{1}$ Department of Cardiology, The First Affiliated Hospital of Jinan University, Guangzhou, China \\ ${ }^{2}$ Interventional Operating Room, The First Affiliated Hospital of Jinan University, Guangzhou, China
}

Email address:

491196769@qq.com (Xuanjing Li),459084901@qq.com (Shengqi Wang),83122801@qq.com (Fan Yang)

${ }^{*}$ Corresponding author

To cite this article:

Xuanjing Li, Shengqi Wang, Fan Yang, Jian Yu, Xianzhen Huang, Wenying Li. Intraoperative Nursing care of Patients Undergoing Transcatheter Aortic Valve Replacement via Femoral Artery Approach. American Journal of Nursing Science. Vol. 10, No. 1, 2021 , pp. 49-54. doi: 10.11648/j.ajns.20211001.19

Received: January 16, 2021; Accepted: January 23, 2021; Published: January 28, 2021

\begin{abstract}
Objective: Intend to explore the main points of intraoperative nursing care of patients with aortic valve stenosis undergoing transcatheter aortic valve replacement through femoral artery approach, summarize the scientific and effective nursing methods in operation. Methods: The intraoperative nursing methods of 9 patients undergoing transcatheter aortic valve replacement through femoral artery approach in our hospital from November 2019 to January 2021 were analyzed retrospectively. These methods include preoperative preparation, transmission of operative consumables, invasive blood pressure monitoring, use of high pressure syringe and temporary pacemaker, nursing of balloon dilatation and valve release, observation and nursing of intraoperative complications, postoperative nursing and so on. Results: The success rate of transcatheter aortic valve replacement was $100 \%$ in 9 patients who underwent transcatheter aortic valve replacement through femoral artery approach. In addition, the average operation time was 194.1 minutes. Besides, 1 case was implanted with middle valve, 1 case was protected by coronary artery, and 1 case was rescued during postoperative transportation. Moreover, no serious operative complications such as valve stent shedding, perivalvular leakage, coronary artery occlusion, stroke and malignant arrhythmia occurred in 9 patients. Conclusion: Full preoperative preparation, familiarity with the whole operation process and key steps, proficiency in the use of high pressure syringe and temporary pacemaker and nursing cooperation during balloon dilatation and valve release are the key to the successful completion of the operation in transfemoral approach transcatheter aortic valve replacement. Through active and effective intraoperative nursing, the operative quality of transfemoral artery approach transcatheter aortic valve replacement can be improved effectively, the operative time can be shortened, and the occurrence of intraoperative complications can be reduced, and the life safety of patients can be guaranteed.
\end{abstract}

Keywords: Aortic Valve Stenosis, Transcatheter Aortic Valve Replacement, Nursing

\section{Introduction}

The incidence of aortic valve stenosis is getting higher and higher with the aging of the population. It is reported that the incidence of aortic valve stenosis is $4 \%$ at the age of 75 and 85 years old and $6 \%$ at the age of 85 [1]. As a consequence, it is a major cardiovascular disease affecting the health of the elderly population [2]. The traditional treatment of aortic valve stenosis is surgery. However, most elderly patients are unable to perform surgery because of poor cardiac function and other diseases such as diabetes. Transcatheter aortic valve replacement (TAVR) has the advantages of minimally invasive, good medium-and long-term effect. In addition, it can be used as an effective treatment for patients with high risk or surgical contraindication of aortic valve stenosis $[3,4]$. In the meanwhile, recent studies have shown that the therapeutic effect of TAVR on medium-and low-risk patients is not inferior to that of traditional surgery $[5,6]$. TAVR via femoral artery approach is the most preferred surgical approach because of its minimally invasive and beneficial to 
the recovery of patients. It is still a high-risk and complex operation compared with traditional interventional surgery though TAVR is becoming more and more safe. Especially for patients with long course of disease, low cardiac ejection fraction, hepatorenal insufficiency and cardiac decompensation aortic valve stenosis, it increases the risk of TAVR operation and the difficulty of intraoperative nursing. The successful and safe completion of TAVR surgery requires not only exquisite medical technology, but also the importance of intraoperative nursing cooperation, which directly affects the quality of surgery and the prognosis of patients. In our hospital, 9 patients with TAVR via femoral artery approach have achieved good surgical results through active and effective intraoperative nursing measures. Now the intraoperative nursing methods are reported as follows.

\section{General Information}

9 cases of TAVR were performed by transfemoral artery approach in our hospital from November 2019 to January 2021. There were 5 males and 4 females and the average age was $74.8 \pm 7.3$ years. In addition, the average operation time was 194.1 minutes. There were 6 cases of aortic valve stenosis, 3 cases of aortic valve insufficiency; 3 cases of coronary heart disease, 1 case of heart failure; 3 cases of diabetes, 2 cases of hypertension; 1 case of middle valve implantation and 1 case of coronary artery protection; and 1 case was rescued during postoperative transport.

\section{Methods}

\subsection{Operation Method}

All the 9 patients were operated by right femoral artery approach under general anesthesia by endotracheal intubation. Bilateral femoral artery, right radial artery and left femoral vein were punctured during the operation, and $6 \mathrm{~F}$ vascular sheath was implanted respectively. In addition, the left femoral vein was punctured and the temporary pacing electrode was placed into the right ventricle for rapid pacing during balloon dilatation and valve release. The left femoral artery was punctured and implanted with $6 \mathrm{~F} 145^{\circ} \mathrm{PIG}$ catheter for intraoperative angiography. Besides, the $6 \mathrm{~F}$ radial artery sheath was inserted into the right radial artery to protect the coronary artery during the operation. The vascular suture device was placed in the right femoral artery, the stiff guide wire was inserted into the left ventricle, the $18 \mathrm{~F}$ vascular sheath was replaced, and the dilated balloon was fed into the dilated aortic valve under rapid pacing (180 beats/min). In addition, the assembled artificial aortic valve was placed at the aortic valve level and was accurately located and released under the guidance of rapid pacing (140 beats/min) and aortic valvulography. Aortic root angiography was performed immediately after release, the placement of the valve was evaluated, the displacement of the valve, perivalvular leakage or occlusion of coronary blood flow were observed, and the function of artificial valve was evaluated by transesophageal ultrasound or transthoracic echocardiography. The vascular sheath of bilateral femoral artery and right radial artery was removed after operation. At the same time, the vascular sheath of left femoral vein and temporary pacing electrode were retained. The preset suture device of the right femoral artery was tightened and bandaged with elastic bandage, the right radial artery was bandaged with inflatable radial artery hemostat. In addition, the left femoral artery was bandaged with elastic bandage, and the patient was transferred to coronary care unit (CCU) or intensive care unit (ICU) for further observation and treatment.

\subsection{Nursing Methods}

\subsubsection{Preoperative Preparation}

\section{(i) Environmental Preparation of Operating Room}

TAVR surgery should be performed in a compound operating room with laminar flow environment. After all, it is beneficial to reduce the occurrence of operation-related infection and emergency surgery when serious complications occur during the operation. In addition, we should open the laminar flow in the operating room 0.5 hours before operation to purify the air, adjust the room temperature to $22{ }^{\circ} \mathrm{C}-25{ }^{\circ} \mathrm{C}$, and the relative humidity to $50 \%-60 \%[7]$. Besides, try to control the number of people in the operating room to less than 8 during the operation, and reduce the flow of personnel. A variety of instruments and equipment should be used during TAVR, and the position of each instrument in the operating room should be placed reasonably to avoid collision with the rotating digital subtraction angiography (DSA) manipulator or hinder the operation of the surgeon and clean and disinfect the surface of the equipment in advance.

\section{(ii) Preparation of Goods and Drugs}

The articles and drugs needed for the operation were prepared one day in advance according to the patient's condition and the operation plan. In addition, the surgical consumables such as artificial aortic valve, transport sheath, $145^{\circ}$ PIG radiography tube, super-slippery guide wire, super-hard guide wire, temporary pacing electrode and vascular suture device were prepared and placed at a fixed point for easy access. Besides, defibrillator, temporary pacemaker, intra-aortic balloon pump (IABP), anesthesia machine, esophageal ultrasound machine, blood transfusion machine and other equipment were prepared and arranged reasonably. Moreover, heparin sodium, epinephrine, dopamine, m-hydroxylamine, atropine and other rescue drugs were prepared 0.5 hours before operation, and frozen saline and contrast agents were prepared one day before operation.

\section{(iii) Patient Preparation}

The basic data of the patients were checked before operation, and the results of cardiac color ultrasound, thoracic and abdominal aorta CTA, electrocardiogram, renal function, blood type, allergy test and so on were checked. Because TAVR surgery takes a long time, and faces mostly elderly patients, the routine operation is in the supine 
position, and the movement should be gentle when helping the patients to put the surgical position. In addition, the decompression pad should be used reasonably to protect the shoulder blades, elbow joint, ankle joint, hip and other parts of the patients from pressure, and keep the joint in the functional position. Besides, expose the anterior chest area, bilateral groin and the surgical site of the right upper limb, cover the quilt in time to keep warm and protect the patient's privacy. And then paste the defibrillator electrode at the bottom and apex of the patient's heart and connect the defibrillator for use during intraoperative cardioversion. Finally, more than two venous channels were established to assist anesthesiologists in induction of intravenous anesthesia and completion of general anesthesia by endotracheal intubation, and indwelling catheter was given to patients after anesthesia.

\subsubsection{Intraoperative Nursing}

\section{(i) Transfer Surgical Consumables}

We checked with the surgeon and opened the prepared TAVR surgical consumables after assisting the surgeon to disinfect the puncture site and spread the towel. When opening the consumables, we paid attention to check the specifications and dimensions, package integrity, sterilization, production validity, etc., strictly aseptic operation to avoid contamination. In addition, we tried to reduce the distance and obstacles between the valve assembly table and the main operating table, and avoid contamination caused by collision between the assembled valve and the transport sheath in the process of transmission.

\section{(ii) Invasive Blood Pressure Monitoring}

2-3 invasive blood pressure monitoring pathways need to be prepared for TAVR surgery. In fact, it can be used to measure the pulse pressure difference of left ventricle and ascending aorta during operation, and to monitor the blood pressure of patients dynamically by anesthesiologist. In addition, we chosen the same type of invasive blood pressure monitoring kit, used heparin saline for full exhaust and zero in the meanwhile. All monitoring kits should be at the same level and at the same level as the patient's heart to reduce measurement errors. In addition, the procedure of setting up the multichannel or monitor is to monitor the invasive blood pressure of 2-3 channels and accurately record the pulse pressure difference before and after the release of the valve. When measuring the pulse pressure difference of left ventricle and ascending aorta during operation, the actual pulse pressure difference should be subtracted from the calculation if there is a difference when different monitoring pathways are used in the same place.

\section{(iii) The Use of High Pressure Syringe}

High-pressure syringe should be used for angiographic examination many times during TAVR. In addition, high-pressure syringe syringe should be used to extract contrast medium to the maximum capacity of $150 \mathrm{ml}$ During the operation. Strict aseptic operation should be performed to avoid contamination of the top of high-pressure syringe syringe during the extraction of contrast medium. The aseptic film cover was used to cover the top of the high-pressure syringe and pushed to the top of the operation bed, and the surgeon used a high-pressure injection connecting tube to connect the top of the syringe before exhaust after the extraction. Covering the aseptic film sleeve is beneficial to avoid the contamination caused by the contact of the guide wire and catheter with the high pressure syringe during the operation. In addition, the injection parameters of the high pressure syringe were adjusted according to the doctor's instructions and were in the state of exposure injection linkage. The injection parameters were routinely set as follows: Before operation, the injection speed of radiography was $20 \mathrm{ml} / \mathrm{s}$, and the total injection volume was $20 \mathrm{ml}$, while during balloon dilatation, the injection speed was $15 \mathrm{ml} / \mathrm{s}$, and the total injection volume was $15 \mathrm{ml}$. When the valve was released, the injection speed was $10 \mathrm{ml} / \mathrm{s}$, and the total injection volume was $10 \mathrm{ml}$. When the valve was released, the injection speed was $20 \mathrm{ml} / \mathrm{s}$, and the total injection volume was $20 \mathrm{ml}$. The pressure of each injection was 800 to $1100 \mathrm{psi}$, and the injection delay was $0 \sim 1 \mathrm{~s}$.

\section{(iv) The Use of Temporary Pacemakers}

Firstly, we checked whether the adjustment switch of the temporary pacemaker was sensitive and whether the battery was sufficient. The pacing function was tested after the pacing electrode was implanted. The pacing heart rate was generally higher than the patient's own heart rate of $10-20$ beats/min, the output voltage was $5 \mathrm{~V}$, and the perception was 2/3 MV. The pacing wire connector was separated from the temporary pacemaker, and the pacing parameters were adjusted after the completion of the test. When rapid pacing was needed, the pacing connector was directly inserted into the pacing connector. The temporary pacemaker could be suspended under the main monitor and the pacemaker electrode was properly fixed to prevent the pacemaker or electrode from falling off due to the movement of the operating bed. In addition, we started or stopped pacing quickly according to the doctor's instructions during the operation to avoid bad pacing affecting balloon dilatation or valve release. The changes of pacing rhythm of patients were closely observed during pacing. If ventricular arrhythmias such as ventricular tachycardia or ventricular fibrillation occur after pacing, defibrillator were used for electrical cardioversion in time. It was necessary to adjust the output voltage to $10 \mathrm{~V}$ and perception to $20.03 \mathrm{mV}$ during rapid pacing with temporary pacemaker. The pacing frequency during balloon dilated valvular release was 160 beats -220 beats/min, during self-expandable valvular release. The pacing rate was generally 100 beats -120 beats $/ \mathrm{min}$. All the 9 patients were treated with self-expanding valve. The pacing rate was set at $140 \mathrm{bpm}$ at the time of valve release. Sinus rhythm was restored in 6 cases and bundle branch block occurred in 3 cases after stopping pacing.

\section{(v) Nursing Care of Patients with Balloon Dilatation}

Balloon dilatation is mainly suitable for patients with 
aortic valve stenosis in order to fully dilate the aortic valve and avoid incarceration or poor release during valve release. A balloon with a diameter of $16 \sim 20 \mathrm{~mm}$ was generally selected. Under rapid pacing, the balloon was quickly and fully dilated, and then the balloon was emptied, and the rapid pacing was stopped when the arterial systolic blood pressure was less than $60 \mathrm{mmHg}$. The total pacing time should be controlled within 15 seconds to avoid serious surgical complications caused by long low perfusion time. The high pressure syringe should be adjusted to multiple linkage injection state, and the pacing frequency of the temporary pacemaker should be set at $180 \mathrm{bpm}$ during balloon dilatation. The lead connection should be quickly inserted into the temporary pacemaker in operation to make it pacing instantly, and the changes of pacing rhythm and arterial blood pressure should be observed when the doctor gives the pacing order. Among the 9 patients, 6 patients with aortic valve stenosis underwent balloon dilatation, of which 4 patients underwent multiple dilatation, and the final dilatation results were satisfactory.

\section{(vi) Nursing Care of Patients with Valve Release}

Valve release should be carried out under the best DSA projection angle. It varies according to the shape of the patient's aortic valve, and mainly refers to the angle measured by multi-slice spiral computed tomography (MSCT) before operation so that the three aortic sinuses are in the same plane. The DSA projection angle should be written on paper and pasted under the main display of the operating room, which is convenient for surgeons to adjust the projection angle before operation. Repeated radiography is needed to confirm the position of the valve in the process of valve release. It ensures that the margin of contrast in the syringe of the high pressure syringe is sufficient and set to the state of multiple injections, and adjusts the pacing frequency of the temporary pacemaker to 140 beats per minute. Quickly start pacing to complete the release of the valve according to the instructions of the surgeon. Among the 9 patients in this group, 8 cases were successfully released at one time, and 1 case had satisfactory results after reimplantation of the second valve due to poor release position of the valve.

\section{(vii) Observation and Nursing of Intraoperative Complications}

The common complications during TAVR include valve stent shedding, perivalvular leakage, coronary artery occlusion and myocardial infarction, stroke, conduction block, vascular complications and so on [8, 9]. Vascular complications are common complications of transfemoral artery approach TAVR, with an incidence of $5.5 \%$ to $20 \%$ [10]. It is mainly related to severe calcification, stenosis or tortuosity of femoral artery, larger diameter of transport sheath and more transvascular approaches. Preoperative evaluation is needed to avoid vascular complications. For patients with small internal diameter of femoral artery, circular calcification of vessel wall, tortuous vessels or deep puncture point, actively cooperate with the surgeon to cut the femoral artery or use vascular suture device for presuture, and evaluate other approaches. When there are vascular complications, cooperate with the surgeon to use balloon occlusion, covered stent implantation or surgical treatment [11]. The surgeon was assisted to apply elastic bandage to bandage the puncture point under local pressure after the operation was completed. We should pay attention to observe the occurrence of hematoma and blood oozing at the puncture site, and touch the pulsation of dorsalis pedis artery and skin temperature after bandaging. In addition, we should observe the skin color of the lower limbs and compare it with the contralateral lower limbs and the situation before operation. All the 9 patients were punctured through the main surgical approach of the right femoral artery and pre-sutured with vascular suture device. The vascular approach was in good condition after operation, and there were no complications such as hematoma, oozing blood, arterial ischemia of lower extremities and so on.

\subsubsection{Postoperative Nursing}

We assisted the surgeon to bandage the bilateral femoral artery, fix the left femoral vein indwelling sheath and the exposed electrode of the temporary pacemaker, measure and record the length of the exposed electrode, and examine the position of the electrode end of the temporary pacemaker in the right ventricle and test the pacing effect before and after fixation after the completion of the operation, avoiding poor pacing caused by electrode displacement after operation. Patients were observed for 30 minutes after awakening from anesthesia, and then transferred to $\mathrm{CCU}$ or ICU, during transport. A defibrillator with monitoring function should be used for continuous vital sign monitoring, and rescue drugs such as atropine, m-hydroxylamine and epinephrine should be carried out to prevent related complications during transport. All the 9 patients were transferred to CCU. And 1 patient after operation, during the transport, the heart rate and blood pressure dropped abruptly, and the consciousness recovered quickly after extrathoracic cardiac compression, and the vital signs returned to normal after intravenous injection of atropine sulfate injection $1 \mathrm{mg}$ and tartrate m-hydroxylamine injection 1mg. The patient was immediately returned to the interventional operating room for angiographic examination of coronary artery blood flow and normal valvular function, and was safely transported to $\mathrm{CCU}$ after continuous observation for 30 minutes.

\section{Results}

The way of transcatheter aortic valve replacement via right femoral artery approach is take measure to the 9 patients in this group, the success rate of the operation is $100 \%$, the average time of this surgery is 194.1 minutes and the average of using contrast media is $195.6 \mathrm{ml}$. One patient was due to poor valve release position, but we got a satisfactory result after putting a Value-in-Value. Another patient suffered from low position of right coronary artery, after the valve was released, it was protected by stent coronary artery through the 
mesh of the valve stent, the angiography which shows the coronary flow is normal and the place of valve is well. One patient experienced sudden drop in heart rate and blood pressure and loss of consciousness during postoperative transport. After timely and effective rescue, vital signs returned to normal and were safely transported to CCU. Through intraoperative meticulous nursing coordination, no serious surgical complications occurred in the 9 patients. All patients recovered and were discharged after continued CCU supportive treatment. The surgeon and the patient are satisfied with the nursing effect.

\section{Discussion}

As a major minimally invasive interventional method for the treatment of aortic valve stenosis [12], compared with surgical valve replacement, TAVR has the advantages of less trauma and faster recovery, and significantly improves the survival time and functional status of patients with aortic valve stenosis [13]. 65 countries around the world have carried out TAVR one after another, with a total of more than 300,000 cases since the first case of TAVR was completed by Cribier [14] in 2002. More than 50 centers in China have carried out the operation one after another, with a total of more than 1000 cases since the academician GE Junbo of Zhongshan Hospital affiliated to Fudan University in Shanghai completed the first TAVR in 2010. At present, TAVR surgery has become more and more mature, which can significantly improve the survival rate of patients with aortic valve stenosis, and has been accepted by more and more patients.

Carrying out TAVR surgery requires a highly professional medical team. In fact, the significance of the team is not limited to the surgical process, but involves evaluation, treatment to rehabilitation guidance [15]. A complete TAVR team should include cardiovascular physicians, cardiovascular surgeons, ultrasound physicians, imaging physicians, anesthesiologists, rehabilitation physicians, imaging technicians, and perioperative nursing teams. Lauck et al [16]. In addition, "team Construction and Operation norms of Transcatheter Aortic Valve replacement" [17] all mentioned the important role of nursing in the team cooperation of TAVR surgery. The members of the nursing team are generally composed of specialist nurses, which are divided into ward specialist nurses, interventional operating room nurses and CCU nurses, who need to be composed of nurses with more than 3 years of working experience. The nurses in the interventional operating room are mainly responsible for intraoperative nursing cooperation, which plays a very important role and directly affects the success or failure of the operation and the prognosis of patients. In addition, nurses in the interventional operating room must be proficient in the types, functions and uses of surgical consumables needed by TAVR, and skillfully use instruments and equipment such as high pressure syringes, temporary pacemakers, IABP counterpulsation pumps, defibrillators, etc. In the meanwhile, it is necessary to manage the environment of the operating room, put the relevant instruments and equipment reasonably, control the flow of personnel, protect the aseptic environment, and supervise the strict implementation of aseptic operation so as to effectively prevent operation-related infection. Moreover, it is necessary to closely observe the process of the operation and the changes of the vital signs of the patients, and cooperate with the doctor in time and effectively to deal with the relevant surgical complications during the operation.

\section{Conclusion}

The nursing during TAVR, especially the nursing cooperation during the operation, requires a high professional level of the nursing staff. It is necessary not only to have a full understanding of the preoperative condition of the patients, but also to skillfully master the specific operation process and the main points of cooperation during the operation, evaluate the possible complications during the operation in time and carry out effective nursing intervention, All of them further ensure the smooth and safe completion of the TAVR operation.

Before surgery, surgical nurses need to prepare the environment of the operating room in advance, reasonably place various instruments and equipment, consumables, drugs, etc., and control the access of personnel involved in the operation, so as to ensure the orderly operation. In TAVR, the most important thing is timely and effective nursing cooperation during balloon dilation and aortic valve release, which requires surgical nurses to master the use method and timing of temporary pacemaker and high pressure syringe skillfully, so as to cooperate with the surgeon to complete the key surgical steps quickly and efficiently.

\section{References}

[1] Walther T, Kempfert J, Rastan A, et al. Transapical aortic valve implantation at 3 years [J]. J Thorac Cardiovasc Surg. 2012, 143 (2): 326-331.

[2] Eveborn G W, Schirmer H, Heggelund G, et al. The evolving epidemiology of valvular aortic stenosis. the Tromso study [J]. Heart. 2013, 99 (6): 396-400.

[3] Eggebrecht H, Vaquerizo B, Moris C, et al. Incidence and outcomes of emergent cardiac surgery during transfemoral transcatheter aortic valve implantation (TAVI): insights from the European Registry on Emergent Cardiac Surgery during TAVI (EuRECS-TAVI)[J]. Eur Heart J. 2018, 39 (8): 676-684.

[4] Gerckens U, Tamburino C, Bleiziffer S, et al. Final 5-year clinical and echocardiographic results for treatment of severe aortic stenosis with a self-expanding bioprosthesis from the ADVANCE Study [J]. Eur Heart J. 2017, 38 (36): 2729-2738.

[5] Thourani V H, Kodali S, Makkar R R, et al. Transcatheter aortic valve replacement versus surgical valve replacement in intermediate-risk patients: a propensity score analysis $[\mathrm{J}]$. Lancet. 2016, 387 (10034): 2218-2225.

[6] Leon M B, Smith C R, Mack M J, et al. Transcatheter or Surgical Aortic-Valve Replacement in Intermediate-Risk Patients [J]. N Engl J Med. 2016, 374 (17): 1609-1620. 
[7] Wang X Z. Analysis of nursing strategy for controlling laminar flow hospital infection [J]. Electronic journal of clinical medical literature. 2017, 4 (75): 14763, 14765.

[8] Genereux P, Head S J, Van Mieghem N M, et al. Clinical outcomes after transcatheter aortic valve replacement using valve academic research consortium definitions: a weighted meta-analysis of 3,519 patients from 16 studies [J]. J Am Coll Cardiol. 2012, 59 (25): 2317-2326.

[9] Kappetein A P, Head S J, Genereux P, et al. Updated standardized endpoint definitions for transcatheter aortic valve implantation: the Valve Academic Research Consortium-2 consensus document (VARC-2) [J]. Eur J Cardiothorac Surg. 2012, 42 (5): S45-S60.

[10] Consensus among Chinese experts on the clinical pathway of transcatheter aortic valve replacement [J]. Chinese Journal of Interventional Cardiology. 2018, 26 (12): 661-668.

[11] De Backer O, Arnous S, Sandholt B, et al. Safety and efficacy of using the Viabahn endoprosthesis for percutaneous treatment of vascular access complications after transfemoral aortic valve implantation [J]. Am J Cardiol. 2015, 115 (8): 1123-1129.

[12] Baumgartner H, Falk V, Bax J J, et al. 2017 ESC/EACTS
Guidelines for the Management of Valvular Heart Disease [J]. Rev Esp Cardiol (Engl Ed). 2018, 71 (2): 110.

[13] Kapadia S R, Leon M B, Makkar R R, et al. 5-year outcomes of transcatheter aortic valve replacement compared with standard treatment for patients with inoperable aortic stenosis (PARTNER 1): a randomised controlled trial [J]. Lancet. 2015, 385 (9986): 2485-2491.

[14] Cribier A, Eltchaninoff $H$, Bash A, et al. Percutaneous transcatheter implantation of an aortic valve prosthesis for calcific aortic stenosis: first human case description [J]. Circulation. 2002, 106 (24): 3006-3008.

[15] Holmes D J, Rich J B, Zoghbi W A, et al. The heart team of cardiovascular care [J]. J Am Coll Cardiol. 2013, 61 (9): 903-907.

[16] Lauck S B, Mcgladrey J, Lawlor C, et al. Nursing leadership of the transcatheter aortic valve implantation Heart Team: Supporting innovation, excellence, and sustainability [J]. Healthc Manage Forum. 2016, 29 (3): 126-130.

[17] Suggestions from Chinese experts on team construction and operation specification of transcatheter aortic valve replacement $[\mathrm{J}]$. Chinese Journal of Interventional Cardiology. 2018, 26 (01): 2-6. 